\title{
Symposium on: Existence of Nash Equilibria in Discontinuous Games
}

\author{
Guilherme Carmona* \\ University of Cambridge
}

September 27, 2010

\begin{abstract}
This paper introduces the symposium on existence of Nash equilibria in discontinuous games.
\end{abstract}

Journal of Economic Literature Classification Numbers: C72

Keywords: Nash equilibrium; discontinuous games.

${ }^{*}$ Faculty of Economics and King's College, University of Cambridge and Faculdade de Economia, Universidade Nova de Lisboa. Address: University of Cambridge, Faculty of Economics, Sidgwick Avenue, Cambridge CB3 9DD, UK; phone: (44) 122333 5225; email: gmc42@cam.ac.uk. 
Game theory studies situations characterized by strategic interaction of several individuals, arising when the well-being of at least one individual depends on what other individuals may do. The analysis of such situations takes two steps. First, they are formally described by a game, which when represented in normal form is a list of players (the individuals), strategies (what these individuals can choose) and payoffs (representing the well-being of individuals associated with different strategies). Second, to the game describing the situation being analyzed, a solution concept is applied to identify strategies with special properties. The interpretation of the resulting equilibrium strategies is that they form reasonable predictions for the problem being studied. Thus, the existence of an equilibrium means that there is at least one reasonable prediction for the problem in hand, and this justifies the importance that the question of existence of equilibrium has historically received.

Nash equilibrium is, perhaps, the most widely used solution concept for normalform games. It was originally defined in Nash (1950), where its existence was established under general conditions. These conditions include, in particular, the continuity of players' payoff functions and Nash's by now classical proof was based on a fixed point theorem (namely, that of Kakutani (1941)).

Motivated by some economic problems that are naturally modeled by games with discontinuous payoff functions, Dasgupta and Maskin (1986), Simon (1987), Simon and Zame (1990), Lebrun (1996), Reny (1999), Carmona (2009), Barelli and Soza (2009), among others, have considerably extended Nash's result and technique. This symposium presents several advances to the literature on existence of Nash equilibrium for games with discontinuous payoff functions that emerged from these papers.

The new results presented in this symposium can be understood in light of the following simple observation: A Nash equilibrium for a game with compact strategy sets exists if (1) the game can be suitable approximated by a sequence of games, (2) each game in this sequence of games has an approximate equilibrium with a vanishing level of approximation and (3) all limit points of every sequence of approximate equilibria, with a level of approximation converging to zero, of these approximating games is a Nash equilibrium of the original game. 
In particular, de Castro (2010) introduces a weak form of reciprocal upper semicontinuity, regularity, and two notions of approximation of games, function approximation and space approximation. ${ }^{1}$ He then shows that if a regular game can be approximated in any those senses and if all approximating games have a Nash equilibrium, then the original game has a Nash equilibrium (under function approximation, the original game needs to be weakly payoff secure as defined in Carmona (2009) and, under space approximation, players' payoff functions need to be lower semicontinuous). In other words, these results provide a sense for condition (1) in such a way that if conditions (1) and (2) hold together with regularity, then condition (3) also holds.

Prokopovych (2010), assuming payoff security (as defined in Reny (1999)) and a weak form of reciprocally upper semicontinuity, shows that the above three-step method can be simplified. ${ }^{2}$ In fact, condition (1) is not needed provided that in condition (2) one uses a suitable notion of approximate equilibrium in the original game. Such notion is obtained by suitably approximating players' value functions from below. This gives us a 2-step method for establishing the existence of Nash equilibria consisting of the following conditions: (2') the game has a sequence of approximate equilibria with a vanishing level of approximation and (3') all limit points of every sequence of approximate equilibria, with a vanishing level of approximation, of the games is a Nash equilibrium. Particularly interesting is the fact that the existence of approximate equilibria (i.e., condition (2')) is established by applying a fixed-point argument in a way similar to that used originally by Nash.

The above two-step method of establishing the existence of Nash equilibria is explicitly presented in Carmona (2010). In particular, the notions of approximate equilibria and of a vanishing level of approximation of such approximate equilibria

\footnotetext{
${ }^{1}$ See Reny (1999) for the definition of reciprocal upper semicontinuity.

${ }^{2}$ It is interesting to note that the weak form of reciprocal upper semicontinuity in Prokopovych (2010), transfer reciprocal upper semicontinuity, is equivalent to regularity. These two notions are also equivalent to the notion of better-reply closeness introduced in Carmona (2010). Furthermore, these notions are equivalent to weak reciprocal upper semicontinuity (as defined in Bagh and Jofre (2006)) at all strategies that are not a Nash equilibrium.
} 
are given a precise meaning and a condition on the game, weak better-reply security, is introduced that allows for an existence result along that two-step method. An important improvement is that these two steps are applied not the the original game but rather to a game obtained by replacing players' payoff functions with better-behaved functions. ${ }^{3}$ In particular, in condition (2') above, it is the better-behaved game (i.e., that with the better-behaved functions) that is required to have approximate equilibria, and in (3'), one has to consider sequences of approximate equilibria of the better-behaved game (but the conclusion is unchanged, i.e., the limit points of such sequences are required to be Nash equilibria of the original game). The importance of this extension of the two-step method consisting of conditions (2') and (3') is that it allows for the existence result of Barelli and Soza (2009) for generalized better-reply secure games, which, in particular extends those of Reny (1999) and Carmona (2009).

Reny (2010) presents a limit result in line of condition (3) above. In his result, the approximation of games is obtained by approximating players' action spaces with finite action spaces. In this way, the result in Reny (2010) is related to the limit result of de Castro (2010) under space approximation. Unlike in de Castro (2010), where the restriction of each player's payoff function to the finite action spaces is related to that player's original payoff function through the assumption of lower semicontinuous payoff functions, no such relationship is assumed in the limit result of Reny (2010). Despite this level of generality, Reny (2010) shows that under general conditions there exists a countable set of pure strategies such that the limit result (i.e., condition (3)) holds provided that the approximating sets of pure strategies eventually include each member of the countable set. ${ }^{4}$

Balder (2010) also establishes a limit result related to condition (3) above. In this limit result, there is a sequence of games and a convergent sequence of Nash equilibria

\footnotetext{
${ }^{3}$ These better-behaved functions are a particular form of an envelope of the original payoff functions: for each players, it is the function that belongs to the class of functions that are both generalized payoff secure (as in Barelli and Soza (2009)) and below the original one and that yields the highest value function within this class.

${ }^{4}$ As requested by Philip Reny, footnote 3 in Reny (2010) should be replaced with the following: "For fixed $B$, the real-valued function of $m$ defined by $m(B)$ can be shown to be measurable."
} 
of those games. However, unlike condition (3), it is not assumed that players' payoff functions are approximating a given profile of payoff functions. Nevertheless, the main result of Balder (2010) shows that a conclusion akin to that in condition (3) holds for some "limit" game. More precisely, it shows that there exist payoff functions, a resolvent payoff profile in the terminology of Balder (2010), that are related to those in the sequence of games and are such that the limit strategy is a Nash equilibria of the game with the resolvent payoff profile.

One of the important consequences of the main theorem in Balder (2010) is that it gives us a limit result for games with an endogenous sharing rule. Games with an endogenous sharing rule, as introduced by Simon and Zame (1990), extend the notion of a normal-form game by describing players' payoffs with a correspondence rather than with a function. In this context, the equilibrium concept (i.e., a solution in the terminology of Simon and Zame (1990)) is a measurable selection of the payoff correspondence and a mixed strategy Nash equilibrium for the normal-form game defined using this selection. The limit result of Balder (2010) provides a limit result for games with an endogenous sharing result. In fact, it shows that if a sequence of solutions is such that each selection in the sequence is continuous and the sequence of strategies is convergent, then there exists a solution such that the strategy is the limit of the sequence of strategies and the selection is closely related to the sequence of selections. As shown in Balder (2010), this limit result can be used to compute and to establish the existence of equilibrium in games with an endogenous sharing rule.

The question of existence of a solution for games with an endogenous sharing rule is also considered in de Castro (2010), more specifically, the relationship between the approaches used in the existence theorems of Reny (1999) and of Simon and Zame (1990). Viewing a normal-form game as a special case of a game with an endogenous sharing rule (namely, one with a singleton-valued payoff correspondence), we can describe the result in de Castro (2010) as showing that for every game that satisfies the conditions in the existence theorem of either Simon and Zame (1990) or Reny (1999), there exists a selection of the payoff correspondence satisfying regularity, i.e., 
his weak notion of reciprocal upper semicontinuity.

\section{References}

Bagh, A., And A. Jofre (2006): "Reciprocal Upper Semicontinuity and Better Reply Secure Games: A Comment," Econometrica, 74, 1715-1721.

BALDer, E. (2010): "An Equilibrium Closure Result for Discontinuous Games," Economic Theory, this issue.

Barelli, P., And I. Soza (2009): "On the Existence of Nash Equilibria in Discontinuous and Qualitative Games," University of Rochester.

Carmona, G. (2009): "An Existence Result for Discontinuous Games," Journal of Economic Theory, 144, 1333-1340.

(2010): "Understanding Some Recent Existence Results for Discontinuous Games," Economic Theory, this issue.

Dasgupta, P., And E. Maskin (1986): "The Existence of Equilibrium in Discontinuous Economic Games, I: Theory," Review of Economics Studies, 53, 1-26.

De Castro, L. (2010): "Equilibria Existence in Regular Discontinuous Games," Economic Theory, this issue.

Kakutani, S. (1941): "A Generalization of Brouwer's Fixed Point Theorem," Duke Mathematical Journal, 8, 457-459.

Lebrun, B. (1996): "Existence of an Equilibrium in First Price Auctions," Economic Theory, 7, 421-443.

NAsh, J. (1950): "Equilibrium Points in N-person Games," Proceedings of the National Academy of Sciences, 36, 48-49.

Prokopovych, P. (2010): "On Equilibrium Existence in Payoff Secure Games," Economic Theory, this issue. 
Reny, P. (1999): "On the Existence of Pure and Mixed Strategy Equilibria in Discontinuous Games," Econometrica, 67, 1029-1056.

(2010): "Strategic Approximations of Discontinuous Games," Economic Theory, this issue.

Simon, L. (1987): "Games with Discontinuous Payoffs," Review of Economic Studies, $54,569-597$.

Simon, L., And W. Zame (1990): "Discontinuous Games and Endogenous Sharing Rules," Econometrica, 58(4), 861-872. 